\title{
Internal Endoconduit Technique during Transcatheter Aortic Valve Implantation
}

\author{
Osamu Sakai ${ }^{1}$ Katsuhiko Oka ${ }^{1}$ Tomoya Inoue $^{1}$ Hitoshi Yaku $^{1}$ \\ ${ }^{1}$ Department of Cardiovascular Surgery, Kyoto Prefectural University \\ of Medicine, Kyoto, Japan \\ Thorac Cardiovasc Surg Rep 2019;8:e5-e7.

\begin{abstract}
Address for correspondence Osamu Sakai, MD, PhD, Department of Cardiovascular Surgery, Kyoto Prefectural University of Medicine, 465, Kajii-cho Kawaramachi Hirokoji, Kamigyo-ku, Kyoto 602-8566, Japan (e-mail: osakai@koto.kpu-m.ac.jp).
\end{abstract}

\author{
Abstract \\ Keywords \\ - valvular surgery \\ - aortic valve \\ - TAVI \\ - peripheral vascular \\ disease
}

The transfemoral approach is the least invasive transcatheter aortic valve implantation (TAVI) approach, but the diameter of the iliofemoral arteries needs to exceed $5 \mathrm{~mm}$. We report a case of limited access transfemoral TAVI by the "internal endoconduit technique," which is well known as a safe and effective dilatational technique for thoracic endovascular aortic repair. Subsequently, we could deliver the device without iliac artery injury and we performed transfemoral TAVI.

\section{Introduction}

Transcatheter aortic valve implantation (TAVI) via the transfemoral approach (TF) has been recognized as the least invasive therapy for high-risk patients with severe aortic stenosis. However, in TF-TAVI, the diameter of the iliofemoral arteries needs to exceed $5 \mathrm{~mm}$ and iliofemoral complications are the most common vascular complications. We report a case of limited access TF-TAVI successfully performed using an "internal endoconduit technique," which is well known as a safe and effective dilatational technique for thoracic endovascular aortic repair (TEVAR).

\section{Case Description}

An 81-year-old woman (body surface area, $1.36 \mathrm{~m}^{2}$ ) was admitted to our hospital with symptomatic severe aortic stenosis. The mean pressure gradient in the aortic valve was $57 \mathrm{~mm} \mathrm{Hg}$, the maximum blood flow velocity was $4.3 \mathrm{~m} / \mathrm{s}$, and the effective orifice area index was $0.29 \mathrm{~cm}^{2} / \mathrm{m}^{2}$. Her comorbidities included left lung cancer (for which curative therapy was planned), diabetes mellitus, and a history of cerebral infarction at the age of 59 years. Magnetic resonance angiography of the cerebral and neck vessels showed stenosis of the left internal carotid siphon. The patient was considered to be at high risk (Euro score 10.05\%, Society of Thoracic Surgeons score 8.80\%) for conventional aortic valve replacement surgery.

received

April 23, 2018

accepted after revision

October 2, 2018
Computed tomography revealed that the anatomies of the aortic annulus and the sinus of Valsalva were appropriate for TAVI. In contrast to this, all the access arteries were defective. The ascending aorta was porcelain, the left subclavian artery was narrow (minimum diameter $<5 \mathrm{~mm}$ with spot calcification at the proximal site), and the bilateral iliac arteries were narrow and extensively calcified (minimum diameter $<5 \mathrm{~mm}$ ) ( - Fig. 1). The ankle brachial pressure index was 0.46 on the right and 0.44 on the left. The transapical approach, which requires left lateral thoracotomy, was considered disadvantageous for the patient, who was scheduled for a left pulmonary operation. Therefore, we selected the TF approach with preballoon dilatation of the right iliac artery. Finally, TF-TAVI using a 26-mm CoreValve Evolut R (Medtronic, Minneapolis, Minnesota, United States) was planned.

The procedure was performed under general anesthesia. Even after dilating the right iliac artery with 6- and 7-mm balloons, neither the $14 \mathrm{~F}$ sheath nor the inline sheath could pass through the iliac artery stenosis. As the $12 \mathrm{~F}$ sheath was the largest sheath that could be passed through the right iliac artery, we decided to use the internal endoconduit technique. A $7-\mathrm{cm}$ long stent graft that was $16 \mathrm{~mm}$ in proximal diameter and $10 \mathrm{~mm}$ in distal diameter (W.L. Gore \& Assoc, Flagstaff, Arizona, United States) and designed for use during infrarenal aortic repair as an iliac extension was deployed from the right common iliac artery to the external iliac artery. The deployed stent graft was extended by $8-\mathrm{mm}$
License terms

(C) 2019 Georg Thieme Verlag KG Stuttgart · New York

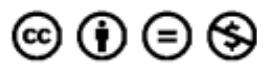




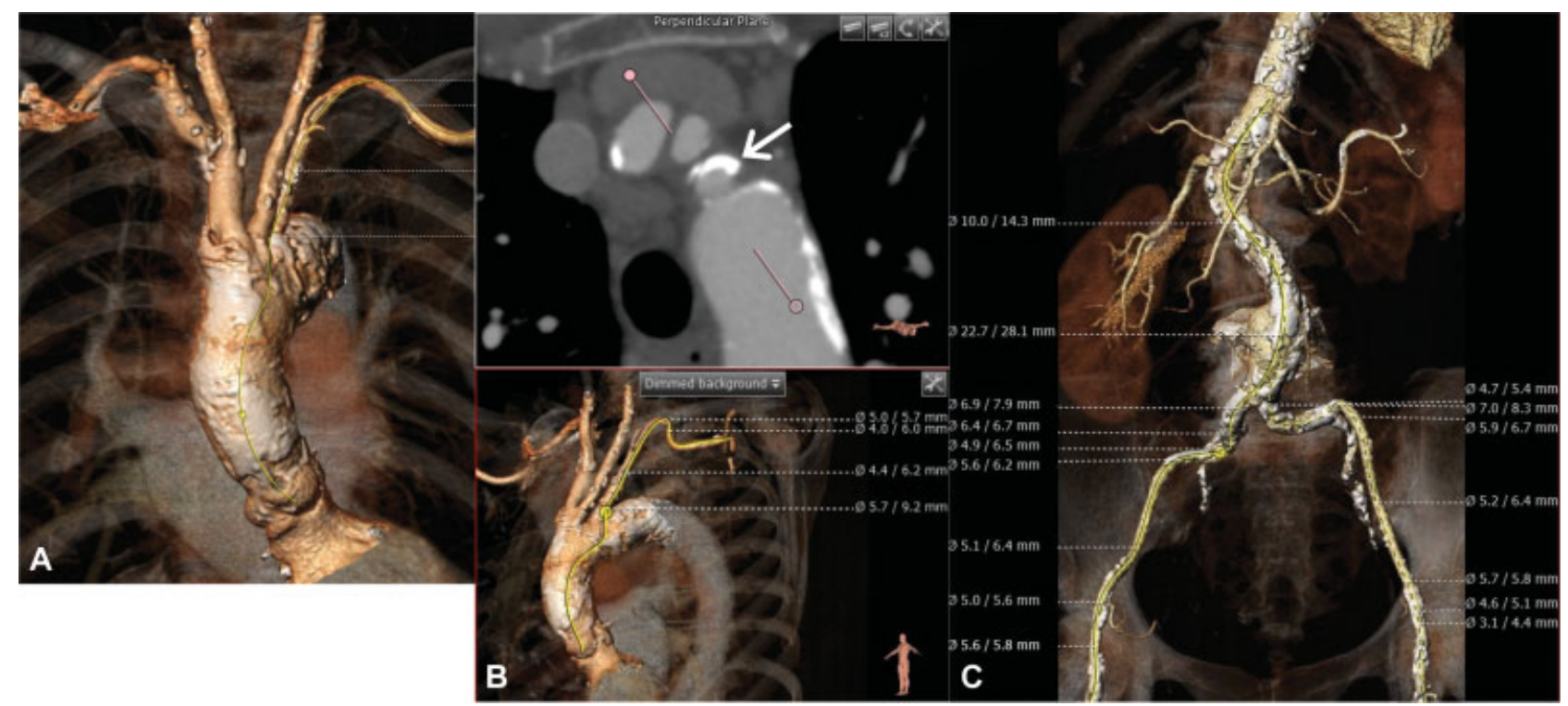

Fig. 1 Computed tomography showing the porcelain ascending aorta (A), narrowing with spot calcification (arrow) of the left subclavian artery (B), and narrowing with extensive calcification of the bilateral iliac arteries (C).

balloon angioplasty without iliac artery rupture. Through the endoconduit, an $18 \mathrm{~F}$ sheath could be passed through the right iliac artery. The delivery system was smoothly inserted into the aortic valve through the $18 \mathrm{~F}$ sheath, and a $26-\mathrm{mm}$ CoreValve was successfully deployed (-Fig. 2).

The postoperative course was uneventful with no iliofemoral complications. The patient was discharged 14 days after the TAVI operation. Postoperative echocardiography revealed an aortic valve mean pressure gradient of $7 \mathrm{~mm} \mathrm{Hg}$, an effective orifice area index of $1.2 \mathrm{~cm}^{2} / \mathrm{m}^{2}$, and a mild paravalvular leak.

\section{Discussion}

The reported rate of major vascular complications ranges from 1.9 to $17.3 \%$ for TF-TAVI. Iliofemoral complications are the most common vascular complications in TF-TAVI. Studies have shown that the combination of an iliofemoral artery diameter smaller than the external sheath size and the concomitant presence of moderate or severe calcification are associated with the highest vascular complication rates. ${ }^{1}$ In TF-TAVI, dissection of the iliofemoral artery has been reported in 1.6 to $7.4 \%$ of patients and rupture of the
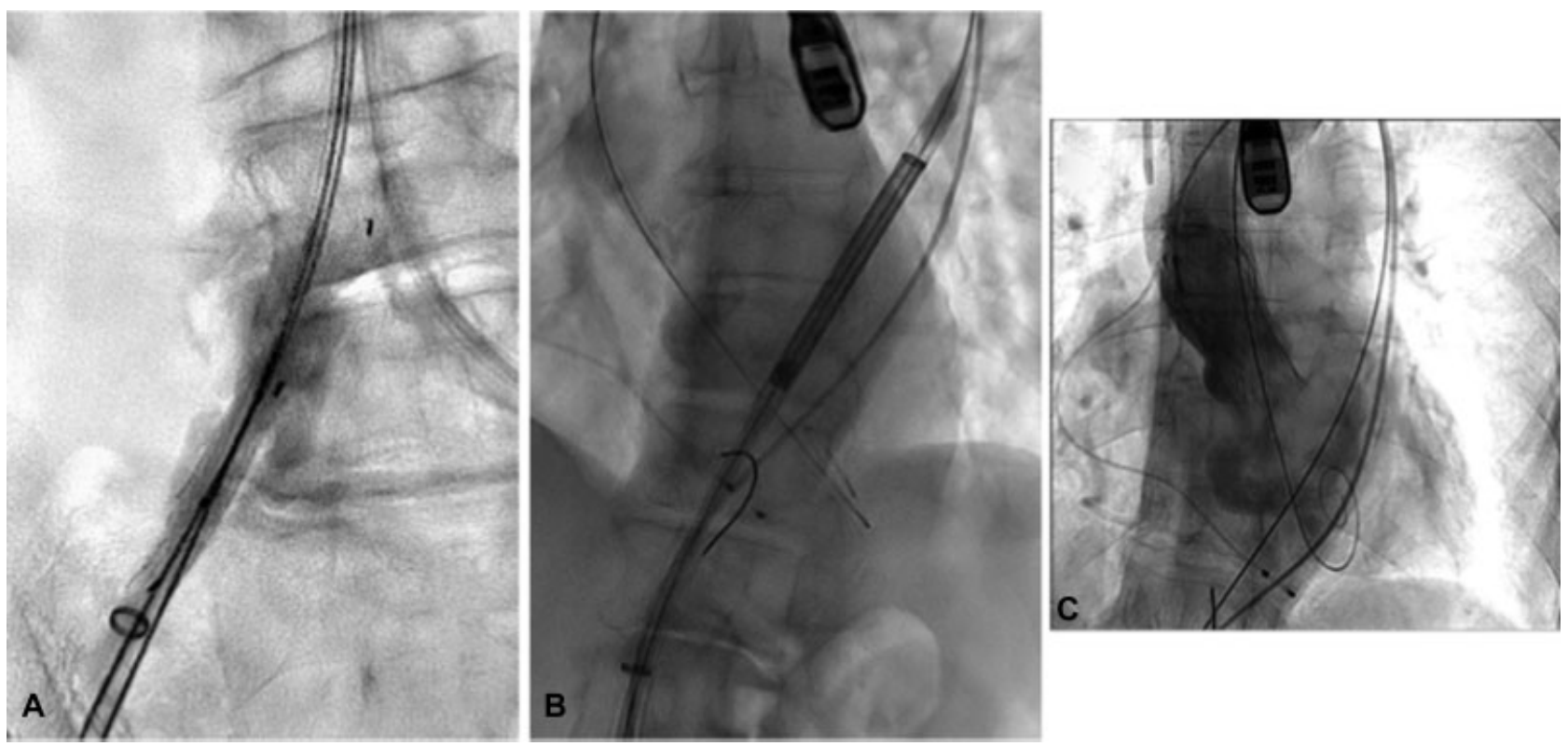

Fig. 2 Angioplasty with an 8-mm balloon within the internal endoconduit (A). An $18 \mathrm{~F}$ sheath was passed through the internal endoconduit, and the delivery system of a 26-mm CoreValve Evolut R (Medtronic, Minneapolis, Minnesota, United States) was passed through the $18 \mathrm{~F}$ sheath (B). The final image shows a well-seated CoreValve (C) and trivial aortic valve leakage. 
iliofemoral artery has been reported in 0.7 to $9.3 \%$ of patients. ${ }^{1,2}$

Transaortic, trans-subclavian, and transapical approaches are options for patients with small iliofemoral arteries and/ or peripheral vascular disease. In our case, the iliofemoral arteries, as well as the left subclavian artery, were small. In addition, all approach arteries had extensive, calcified atherosclerotic disease. The transapical approach requires lateral thoracotomy and myocardial injury, which sometimes leads to surgical repair of the apex of the left ventricle. Our patient had left lung cancer as a complication, and she was scheduled to undergo a pulmonary operation via left-sided thoracoscopy. Thus, the transapical approach would be disadvantageous because of the planned lung operation.

Therefore, we planned TF-TAVI after angioplasty of the right iliac artery. Simpler methods, such as standard angioplasty or serial dilatation with hydrophilic dilators, often allow the passage of delivery sheaths. However, aggressive angioplasty often leads to iliac artery dissection or rupture. Peterson initially described the internal endoconduit approach as an alternative stent graft delivery technique using TEVAR. ${ }^{3}$ The internal endoconduit technique allows aggressive balloon dilatation of the long segments of iliofemoral stenosis without the risk of vessel rupture. This technique has the advantage of requiring only femoral access. Using the internal endoconduit approach for TEVAR, patients can be treated via standard femoral artery access, even in the presence of significant iliac occlusive disease or other adverse iliac anatomies. ${ }^{4}$ This approach is reported to be safe and effective and is associated with low rates of early mortality and late iliofemoral complications. ${ }^{5}$ Recently, Murakami et al reported this technique using an Endurant II stent graft iliac extension (Medtronic Vascular, Inc., Santa Rosa, California, United States) and Fluency Plus stent graft (CR Bard, Inc., Murray Hill, New Jersey, United States) for TF-TAVI. ${ }^{6}$

We applied this internal endoconduit technique to TFTAVI with good results. The delivery system was inserted safely and smoothly. There were no perioperative iliofemoral complications. The internal endoconduit technique may be considered a suitable delivery route for selected patients requiring TAVI.

Conflicts of Interest

Author discloses a financial relationship with Japan Gore, Japan.

\section{References}

1 Toggweiler S, Leipsic J, Binder RK, et al. Management of vascular access in transcatheter aortic valve replacement: part 2: vascular complications. JACC Cardiovasc Interv 2013;6(08):767-776

2 Hayashida K, Lefèvre T, Chevalier B, et al. Transfemoral aortic valve implantation new criteria to predict vascular complications. JACC Cardiovasc Interv 2011;4(08):851-858

3 Peterson BG, Matsumura JS. Internal endoconduit: an innovative technique to address unfavorable iliac artery anatomy encountered during thoracic endovascular aortic repair. J Vasc Surg 2008; 47(02):441-445

4 Kpodonu J, Rodriguez-Lopez JA, Ramaiah VG, Diethrich EB. "Cracking and paving": a novel technique to deliver a thoracic endograft despite ilio-femoral occlusive disease. J Card Surg 2009; 24(02):188-190

5 van Bogerijen GHW, Williams DM, Eliason JL, Dasika NL, Deeb GM, Patel HJ. Alternative access techniques with thoracic endovascular aortic repair, open iliac conduit versus endoconduit technique. J Vasc Surg 2014;60(05):1168-1176

6 Murakami T, Takahashi Y, Nishimura S, et al. Endoconduit for transcatheter aortic valve implantation. Ann Thorac Cardiovasc Surg 2018. Doi: 10.5761/atcs.cr.17-00204 